\title{
Analysis of tau post-translational modifications in rTg4510 mice, a model of tau pathology
}

\author{
Lixin Song ${ }^{1,4}$, Sherry X Lu', Xuesong Ouyang ${ }^{1,5}$, Jerry Melchor²,6 Julie Lee ${ }^{1}$, Giuseppe Terracina ${ }^{1}$, Xiaohai Wang ${ }^{3}$,
} Lynn Hyde ${ }^{1}$, J Fred Hess ${ }^{1}$, Eric M Parker ${ }^{1 *}$ and Lili Zhang ${ }^{1,7^{*}}$

\begin{abstract}
Background: Microtubule associated protein tau is the major component of the neurofibrillary tangles (NFTs) found in the brains of patients with Alzheimer's disease and several other neurodegenerative diseases. Tau mutations are associated with frontotemperal dementia with parkinsonism on chromosome 17 (FTDP-17). rTg4510 mice overexpress human tau carrying the P301L FTDP-17 mutation and develop robust NFT-like pathology at 4-5 months of age. The current study is aimed at characterizing the rTg4510 mice to better understand the genesis of tau pathology and to better enable the use of this model in drug discovery efforts targeting tau pathology.
\end{abstract}

Results: Using a panel of immunoassays, we analyzed the age-dependent formation of pathological tau in rTg4510 mice and our data revealed a steady age-dependent accumulation of pathological tau in the insoluble fraction of brain homogenates. The pathological tau was associated with multiple post-translational modifications including aggregation, phosphorylation at a wide variety of sites, acetylation, ubiquitination and nitration. The change of most tau species reached statistical significance at the age of 16 weeks. There was a strong correlation between the different post-translationally modified tau species in this heterogeneous pool of pathological tau. Total tau in the cerebrospinal fluid (CSF) displayed a multiphasic temporal profile distinct from the steady accumulation of pathological tau in the brain. Female rTg4510 mice displayed significantly more aggressive accumulation of pathological tau in the brain and elevation of total tau in CSF than their male littermates.

Conclusion: The immunoassays described here were used to generate the most comprehensive description of the changes in various tau species across the lifespan of the rTg4510 mouse model. The data indicate that development of tauopathy in rTg4510 mice involves the accumulation of a pool of pathological tau that carries multiple posttranslational modifications, a process that can be detected well before the histological detection of NFTs. Therapeutic treatment targeting tau should therefore aim to reduce all tau species associated with the pathological tau pool rather than reduce specific post-translational modifications. There is still much to learn about CSF tau in physiological and pathological processes in order to use it as a translational biomarker in drug discovery.

Keywords: Alzheimer's disease, Neurodegeneration, Tau, Tau aggregation, Tau phosphorylation, Tau acetylation, rTg4510, CSF tau

\section{Background}

Microtubule associated protein tau is expressed primarily in neurons and plays an important role in axonal transport [1]. Abnormal accumulation of tau in the brain of patients with Alzheimer's disease (AD) leads to the

\footnotetext{
* Correspondence: eric.parker@merck.com; Ilzh@icloud.com

${ }^{1}$ Department of Neuroscience, Merck Research Laboratories, Kenilworth, NJ, USA

${ }^{7}$ Current address: Department of Neuroscience, Novartis Institute for Biomedical Research, Cambridge, MA, USA

Full list of author information is available at the end of the article
}

formation of neurofibrillary tangles (NFTs), which together with $\beta$-amyloid plaques are the two pathological hallmarks of the disease [2,3]. While plaques can be found in post-mortem brains from people without significant clinical AD symptoms, the presence of NFTs correlates well with neuronal cell death and the loss of cognitive functions. In addition, NFTs are also found in the brains of patients with several other neurodegenerative diseases that lack amyloid pathology [2]. Understanding the development of tau pathology, also known as tauopathy, should provide important insights into the

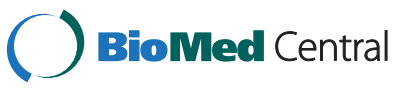

(c) 2015 Song et al.; licensee BioMed Central. This is an Open Access article distributed under the terms of the Creative Commons Attribution License (http://creativecommons.org/licenses/by/4.0), which permits unrestricted use, distribution, and reproduction in any medium, provided the original work is properly credited. The Creative Commons Public Domain Dedication waiver (http://creativecommons.org/publicdomain/zero/1.0/) applies to the data made available in this article unless otherwise stated. 
etiology of $\mathrm{AD}$ and other neurodegenerative diseases, and into the development of therapeutic strategies targeting this pathway.

Human tau exists as six alternatively spliced isoforms, with its C-terminal half containing either 3 or 4 repeats of the microtubule binding domain (designated as either $3 R$ or $4 R$ tau). In adult brain under normal physiological conditions, the $3 R$ and $4 R$ tau are present in about a 1:1 ratio. Pathological tau in $\mathrm{AD}$ brain is hyperphosphorylated and forms insoluble aggregates that eventually develop into NFTs [2]. This observation has led to the hypothesis that abnormal phosphorylation plays a major role in the disease process. In addition to phosphorylation, tau undergoes multiple posttranslational modifications such as acetylation, nitration, ubiquitination, etc. $[4,5]$. Moreover, tau is also present in cerebral spinal fluid (CSF) and tau in CSF is elevated in AD patients long before the clinical symptoms of the disease are manifest [6]. These findings suggest that the development of tauopathy is dependent on far more than just hyperphosphorylation. The biological and pathological processes involved in tau post-translational modifications and CSF tau production are largely unknown.

The identification of tau mutations associated with frontotemporal dementia with parkinsonism on chromosome 17 (FTDP-17) [7,8] establishes the pathogenic role of tau in mediating neurodegeneration and cognitive decline. FTDP-17 mutations affect the tau microtubule binding or alternative splicing in favor of the $4 \mathrm{R}$ form of tau (reviewed by [1]). Many transgenic animal models have been generated to explore the mechanism of tauopathy development. Transgenic models expressing human tau with missense FTDP-17 mutations display more robust NFT formation compared to those that express wild-type human tau [9]. One of the models, the rTg4510 mouse, overexpresses a human tau transgene carrying a P301L mutation, with transgene expression driven by the $\mathrm{Ca}^{2+}$-calmodulin kinase II promoter [10]. NFT staining can be detected in rTg4510 mice as early as 5-6 months of age and, like NFT pathology in $\mathrm{AD}$, is restricted to the forebrain structures such as hippocampus and cortex. This model also develops neuronal cell loss, brain atrophy and cognitive decline reminiscent of that seen in $\mathrm{AD}$ patients $[10,11]$, making it an attractive model to investigate disease mechanisms and test potential therapeutic agents.

Here we report the development of a comprehensive panel of sandwich immunoassays to study the tauopathy in rTg4510 mice. The sensitivity and quantitative nature of these assays enabled us to investigate the correlations between different tau species over the lifespan of the rTg4510 mice, thereby providing important information on the properties of pathological tau and the time course of its development in this model. These findings will guide the effective use of rTg4510 mice in studies of the basic biology of $\mathrm{AD}$ and other tauopathies, and in the assessment of potential treatments for these conditions.

\section{Results}

To study changes in tau during disease progression in rTg4510 mice, we separated the brain homogenates into soluble and insoluble fractions by a simple two step centrifugation process based on a previously reported study [12]. Western blots probed with HT7 and tau-12 antibodies, which recognize epitopes independent of phosphorylation and detect total tau, revealed an age-dependent decrease of total tau in the soluble fraction and an age-dependent increase of total tau in the insoluble fraction. Tau in the insoluble fraction also underwent a transition from a $55 \mathrm{kD}$ species to a higher molecular weight $64 \mathrm{kD}$ species as the animals aged (Figure 1A). There were two distinct profiles of phosphorylated tau (p-tau) changes during disease progression in the $\mathrm{rTg} 4510$ mice. One type of $\mathrm{p}$-tau, as detected by antibodies AT8, PHF6, AT180, PHF13 and pS409, was enriched in the $64 \mathrm{kD}$ form in the insoluble fraction and increased in the insoluble fraction as the animals aged (Figure 1B). The second type of p-tau, as detected by antibodies AT270, pS400, pS404 and pS412, displayed a profile similar to that of total tau, i.e., a decrease in the soluble fraction, an increase in the insoluble fraction and a transition from a $55 \mathrm{kD}$ to a $64 \mathrm{kD}$ species in the insoluble fraction as the animals aged (Figure 1C). These data are consistent with observations from other groups and demonstrate an age-dependent development and accumulation of a $64 \mathrm{kD}$ species of pathological tau that is enriched in the insoluble fraction of brain extracts and correlates with the development of tauopathy, neuronal cell loss, brain atrophy and cognitive impairment [10].

Next we focused on the change of tau in the insoluble fraction in which the pathological $64 \mathrm{kD}$ tau species was enriched. A panel of sensitive sandwich immunoassays was developed using AlphaLISA technology (PerkinElmer) to analyze different tau species during the progression of tauopathy in $\mathrm{rTg} 4510$ mice (Table 1). An age-dependent accumulation of both total tau and aggregated tau was observed in the insoluble fraction (Figure 2A and 2B). It is noteworthy that large inter-animal variations in both total and aggregated tau were observed within each age group, particularly at older ages where tauopathy was progressing rapidly and significant amounts of tau were accumulating in the insoluble fraction. The various species of p-tau studied also showed an age-dependent increase in the insoluble fraction, including p-tau species detected by PHF6 (conformation around pT231; Figure 3A), AT8 (pS202/T205; Figure 3B), pS262, pS400, AT180 (pT231), PHF13 (pS396), AT100 (pS212/pT214) and pS409 (Additional file 1: Figure S1). We also developed an immunoassay for global tau phosphorylation using a pan anti-phosphothreonine antibody and showed that, similar to other p-tau species, pThr global tau phosphorylation was also elevated in the insoluble fraction (Figure 3C). For most of the tau species in the soluble fraction, the age-dependent increase reached statistical 


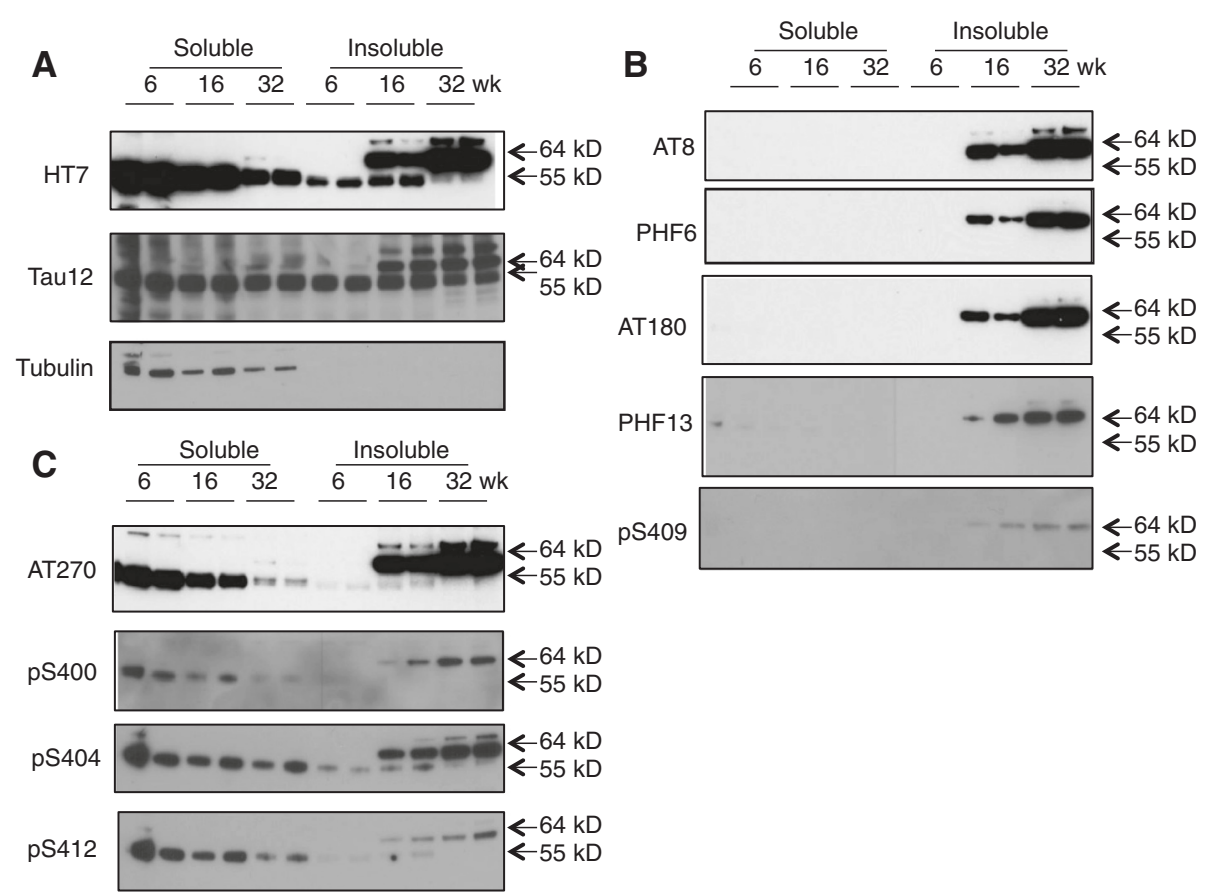

Figure 1 Western blot analysis of various tau species in brain lysates from Tg4510 mice of different ages. Soluble and insoluble fractions of brain homogenates were prepared as described in Methods from rTg4510 mice that were 6,16 or 32 weeks of age. Ten $\mu \mathrm{g}$ of protein from each fraction was analyzed by probing Western blots with the specified antibodies. The position of the $55 \mathrm{kD}$ and $64 \mathrm{kD}$ species of tau are indicated. a-Tubulin was used as a loading control. A. Total tau detected by two different antibodies (HT7 and Tau12). B. P-tau species enriched in $64 \mathrm{kD}$ tau in the insoluble fraction. C. P-tau species present in both the $55 \mathrm{kD}$ species in the soluble fraction and in the $64 \mathrm{kD}$ species in the insoluble fraction.

significance at 16 weeks as compared to 8 weeks of age (one-way ANOVA, $\mathrm{p}<0.05$, Figures 2 and 3 , Additional file 1: Figure S1).

A further analysis revealed that despite the large variations between animals, the levels of all p-tau species showed strong correlation with the levels of the p-tau species recognized by PHF6, including AT8 $\left(R^{2}=0.9, p<0.0001\right.$, Figure 4A), pThr global phosphorylation $\left(\mathrm{R}^{2}=0.89\right.$, $\mathrm{p}<0.0001$, Figure 4B), pS262 $\left(\mathrm{R}^{2}=0.82, \mathrm{p}<0.0001\right), \mathrm{pS} 400$ $\left(R^{2}=0.88, \quad p<0.0001\right), \quad$ AT180 $\quad\left(R^{2}=0.93, \quad p<0.0001\right)$, PHF13 $\left(R^{2}=0.9, p<0.0001\right)$, AT100 $\left(R^{2}=0.94, p<0.0001\right)$ and pS409 $\left(\left(\mathrm{R}^{2}=0.8, \mathrm{p}<0.0001\right.\right.$, Additional file 2: Figure $\left.\mathrm{S} 2\right)$. In addition, a significant correlation was observed between PHF6 tau and tau aggregates $\left(\mathrm{R}^{2}=0.80, \mathrm{p}<0.0001\right.$, Figure $4 C)$ and between PHF6 tau and total tau $\left(R^{2}=0.69\right.$, $\mathrm{p}<0.0001$, Figure 4D) in the insoluble fraction.

Tau acetylation has recently been reported to be increased in the brains of tau transgenic mice and in the brains of AD patients [13,14]. Western blots probed with Ac-280 showed this tau species acetylated at Lys280 migrated with the $55 \mathrm{kD}$ tau species in the soluble fraction from young rTg4510 mice but shifted to the $64 \mathrm{kD}$ species in the insoluble fraction as tauopathy progressed (Figure 5A). An immunoassay to assess global tau acetylation was developed that utilized a pan anti-acetylated lysine antibody in combination with antibody HT7. This immunoassay confirmed that the global tau acetylation was much enriched in the insoluble fraction as compared to the soluble fraction. The acetylated tau was elevated as the rTg4510 animals aged and the change reached statistical significance at the age of 14-weeks as compared to 8 -weeks ( $\mathrm{p}<0.05$, one-way ANOVA, Figure $5 \mathrm{~B}$ ). Interestingly, acetylated tau also showed a strong correlation with PHF6 tau in the insoluble fraction $\left(R^{2}=0.76, p<\right.$ 0.0001 , Figure $5 \mathrm{C}$ ), suggesting that both modifications are present in the same pool of tau that contribute to tauopathy and neurodegeneration in this model.

Next we examined ubiquitination and tyrosine nitration of tau in $\mathrm{rTg} 4510$ mice, both of which have also been shown to be associated with NFT pathology [15-17]. As seen with multiple p-tau species and acetylated tau, both ubiquitinated tau (ub-tau) and tau nitrated at tyrosine 29 (nY29 tau) were enriched in the insoluble fraction and showed agedependent increases in rTg4510 mice (Figure 6A and B). We did not detect either ub-tau or nY29 tau in the soluble fraction using the assays described here (data not shown). Compared to 8-week old mice, the changes of ub-tau and nY29 tau reached statistical significance at the age of 12 and 16-weeks, respectively ( $\mathrm{p}<0.05$, one-way ANOVA, Figure $6 \mathrm{~A}$ and $\mathrm{B})$. Again, the change of both tau species 


\begin{tabular}{|c|c|c|}
\hline Tau Species & Biotin-donor & Acceptor \\
\hline Total tau & Tau12 & HT7 \\
\hline Tau aggregates & HT7 & HT7 \\
\hline p231 & HT7 & PHF6 \\
\hline pT202/205 & HT7 & AT8 \\
\hline Global p-tau & HT7 & anti-pThr \\
\hline pT231 & HT7 & AT180 \\
\hline pT181 & HT7 & AT270 \\
\hline $\mathrm{pS} 212 / \mathrm{T} 214$ & HT7 & AT100 \\
\hline pS262 & HT7 & pS262 \\
\hline pT396 & HT7 & PHF13 \\
\hline pS400 & HT7 & pS400 \\
\hline pS404 & HT7 & pS404 \\
\hline pS409 & HT7 & pS409 \\
\hline pS412 & HT7 & pS412 \\
\hline Ac-Lys 280 & HT7 & Anti-Ac-Lys 280 \\
\hline Ac-tau & HT7 & anti-acetyl-Lys \\
\hline pY-tau & HT7 & anti-p-tyrosine \\
\hline ub-tau & HT7 & anti-ubiquitin \\
\hline nY29 tau & HT7 & anti-nY29 \\
\hline CSF total tau & HT7 & BT2 \\
\hline
\end{tabular}

correlated strongly with the change of PHF6 tau during the development of tauopathy $\left(R^{2}=0.70, p<0.0001\right.$ for $n Y 29$ and $R^{2}=0.94, p<0.0001$ for ub-tau, Figure $6 C$ and $D$ ).

Similar to the various tau species comprising pathological tau in the brain, there was a large inter-animal variation in CSF tau as rTg4510 mice age (Figure 7A). However, unlike the steady accumulation of total tau and various p-tau species in brain as rTg4510 mice age (e.g. as illustrated by PHF6 tau levels in Figure 7B), levels of total tau in CSF of rTg4510 mice changed in a multi-phasic pattern during aging. CSF total tau consistently decreased from 6 to $8-12$ weeks of age, although the decrease was not statistically significant. Subsequently, CSF total tau progressively increased, with the increase reaching statistical significance at 16 weeks of age and beyond (one-way ANOVA, $\mathrm{p}<0.05$ ). After reaching a peak at 20 weeks of age, CSF total tau then showed a steady decline that was statistically significantly from 24-32 weeks of age ( $\mathrm{p}<0.05$, one-way ANOVA) (Figure $7 \mathrm{~B}$ ).

When gender difference was assessed, female $\operatorname{Tg} 4510$ mice had an overall higher level of total tau (main effect of sex, $F(1,166)=19.43, \mathrm{p}<0.00010)$, tau aggregates (main effect of sex, $\mathrm{F}(1,170)=6.59, \mathrm{p}<0.02$ ) and PHF6 tau (main effect of sex, $\mathrm{F}(1,172)=18.78, \mathrm{p}<0.0001$ ) as the mice aged; however, rTg4510 mice of both genders reached similar peak values at 30-32 weeks of age
(Figure 8A-C). Female Tg4150 mice also had higher CSF total tau levels during aging as compared to male mice [main effect of sex, $\mathrm{F}(1,197)=21.24, \mathrm{p}<0.0001$ ], but eventually similar levels of CSF total tau were observed in females and males at ages $>24$ weeks (Figure 8D).

\section{Discussion}

rTg4510 mice express human tau with the P301L mutation and provide many advantages to study tauopathy, including a rapid onset of NFT formation and a brain distribution of NFTs mirroring that seen in AD patients. The development of tauopathy in this model has been well characterized in several studies [10-12,18]. We report here using an immunoassay platform to analyze different tau species in $\mathrm{rTg} 4510$ mouse brain. The higher throughput of this platform enabled us to study larger groups of animals to mitigate the large inter-animal variations in the time course and extent of tauopathy in this model. The sensitivity and the quantitative nature of these assays also allowed us to more precisely investigate the relationships between different features of tauopathy.

One of the characteristic features of NFT pathology in the brains of $\mathrm{AD}$ patients is the accumulation of hyperphosphorylated tau aggregates [2]. Many of the serine and threonine residues in tau have been reported to be phosphorylated in the brains of $A D$ patients [4]. The hyperphosphorylation of tau is associated with a transition of tau from a predominantly soluble, lower molecular weight species to a predominantly insoluble, higher molecular weight species, a transition that is thought to be intimately associated with the development of tauopathy and neurodegeneration [2]. Consistent with previous reports $[10,19]$, our data showed that as rTg4510 mice age, tau progressively transitions from a $55 \mathrm{kD}$ species found predominantly in the soluble fraction of brain homogenates to a $64 \mathrm{kD}$ species found predominantly in the insoluble fraction. Interestingly, some p-tau species are present in both the soluble and insoluble fractions of rTg 4510 brain homogenates, while other $\mathrm{p}$-tau species are primarily present in the insoluble fraction. However, both are present in the insoluble, $64 \mathrm{kD}$ form of tau that is thought to be associated with the development of tauopathy (Figure 1). Thus, the $64 \mathrm{kD}$ tau cannot be said to comprise only one or even a few p-tau species, but rather encompasses the global phosphorylation of tau at all epitopes studied. These data also suggest that the accumulation of $64 \mathrm{kD}$ tau in the insoluble fraction can be used as a surrogate marker to monitor tauopathy development and that a treatment reducing pathological tau formation should lead to a correlative reduction of all tau species associated with tauopathy. Although NFT histology is typically first observed at 4-5 months of age, our biochemical analysis points to a steady accumulation of PHF6 and other pathological forms of tau starting from the young age of 6-8 weeks, with the 

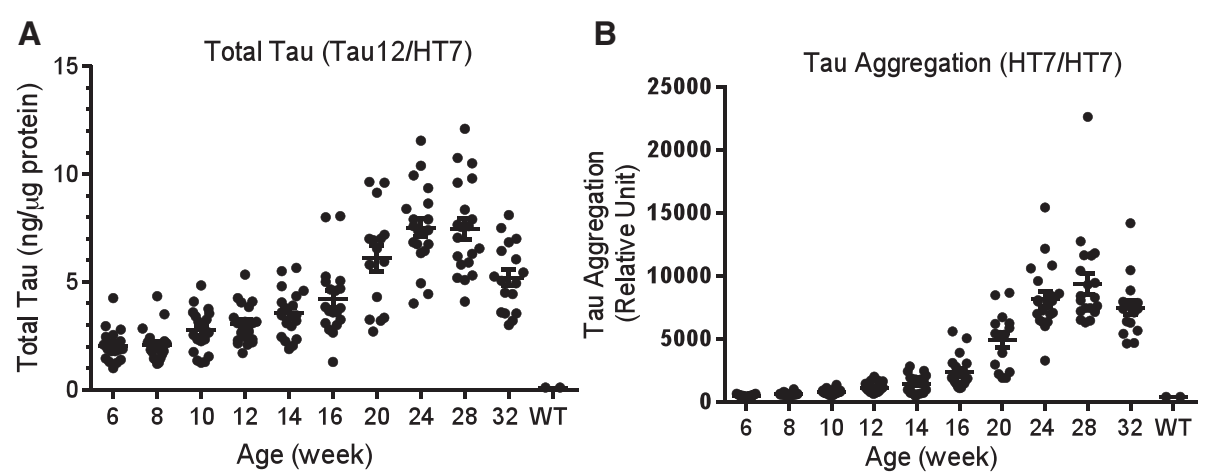

Figure 2 Age-dependent change of total tau and tau aggregates in the brain insoluble fraction of rTg4510 mice. Brain insoluble fractions from animals 6-32 weeks of age ( $n=18-20$ per group) were analyzed using AlphaLISA based immunoassays. A. Total tau measured in an immunoassay using biotin-Tau12 for capture and HT7-conjugated acceptor beads for detection. Twenty ng of protein from the insoluble fractions were used per assay. Recombinant tau (Millipore) was used to generate a standard curve for quantification. B. Tau aggregates measured in an immunoassay using HT7 as both capture and detection antibody. Two hundred ng of protein from the insoluble fractions were used in each assay. The Y-axis is the relative fluorescence readout from Envision (PerkinElmer). Compared to 8-week old mice, total tau and tau aggregate changes reached statistical significance after 14-, and 16-weeks of age, respectively $(p<0.05$, one-way ANOVA).

changes reaching statistical significance by around 16-20 weeks. This finding points to the possibility for the effective use of the rTg4510 model to assess prophylactic treatments targeting tauopathy at ages as early as $6-8$ weeks and for durations of treatment as short as $8-10$ weeks.

In addition to phosphorylation, several additional posttranslational modifications are also associated with pathological tau $[4,5]$. Like many species of p-tau, several other post-translationally modified forms of tau increased in the insoluble fraction of brain homogenates as rTg4510 mice aged, including tau aggregates, ac-tau, ub-tau and nY29 tau. Acetylated tau also transitioned from a predominantly soluble $55 \mathrm{kD}$ species to an insoluble $64 \mathrm{kD}$ species in rTg4510 brain homogenates as the animals aged (Figure 6A). These data provide the first comprehensive characterization of the behavior of these more recently described post-translationally modified forms of tau in the rTg4510 mouse model.
Our data demonstrated a close correlation between all the post-translationally modified species of tau examined in this study (Figures 4, 5 and 6, Additional file 2: Figure S2), suggesting these modifications are not separate and unrelated events but may collectively contribute to the disease process. Pathological tau thus represents a heterogeneous pool of mis-folded proteins encompassing tau phosphorylated at multiple epitopes as well as multiple additional post-translationally modified tau species. These post-translational modifications may occur more or less simultaneously as tauopathy develops and it is difficult to distinguish which one(s) (aggregation, phosphorylation, acetylation, etc.) may be the primary driver of the development of pathological tau. Further investigation in these areas is critical to understand the disease mechanism in the rTg4510 model and assess therapeutics targeting tauopathy.

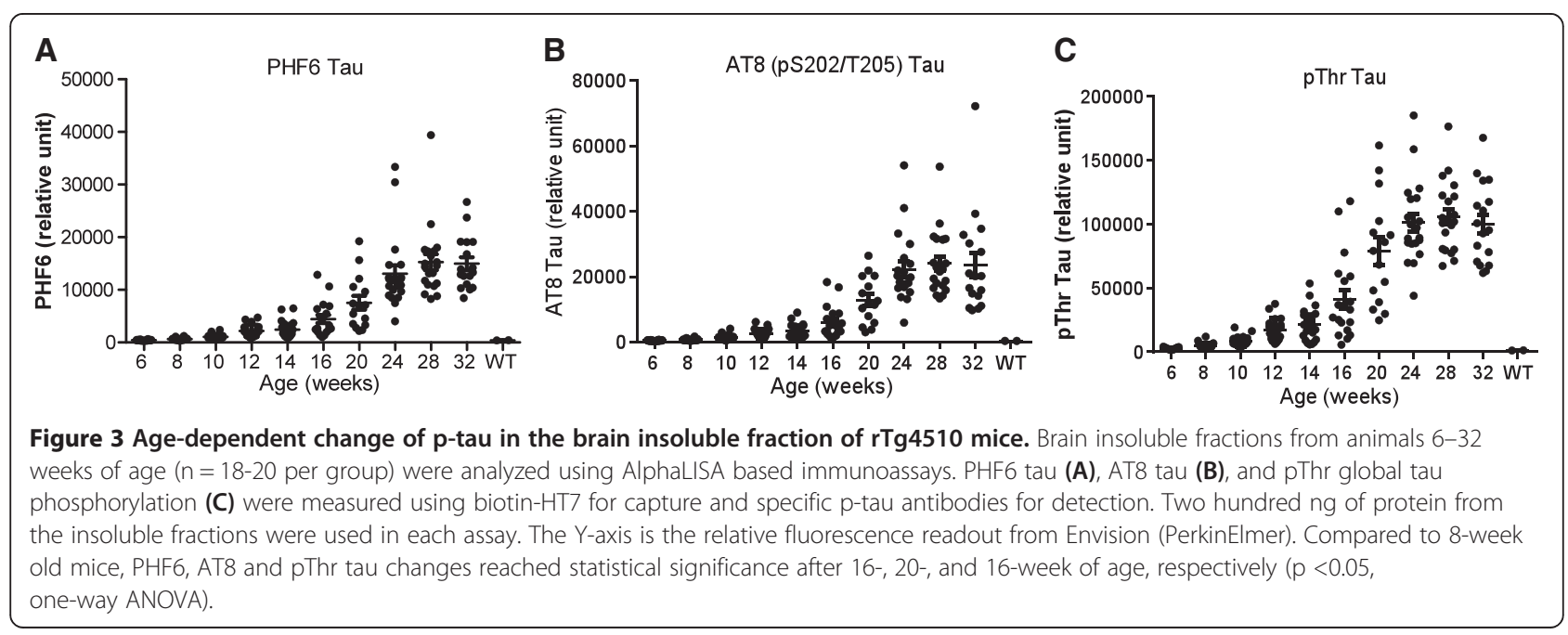



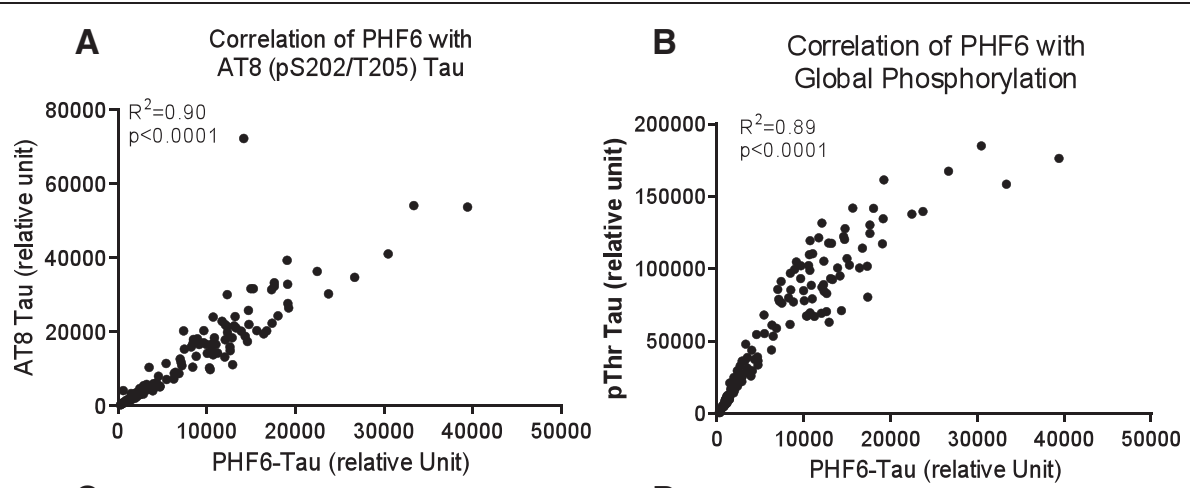

C Correlation of PHF6 with

D tau aggregates

Correlation of PHF6 with total tau
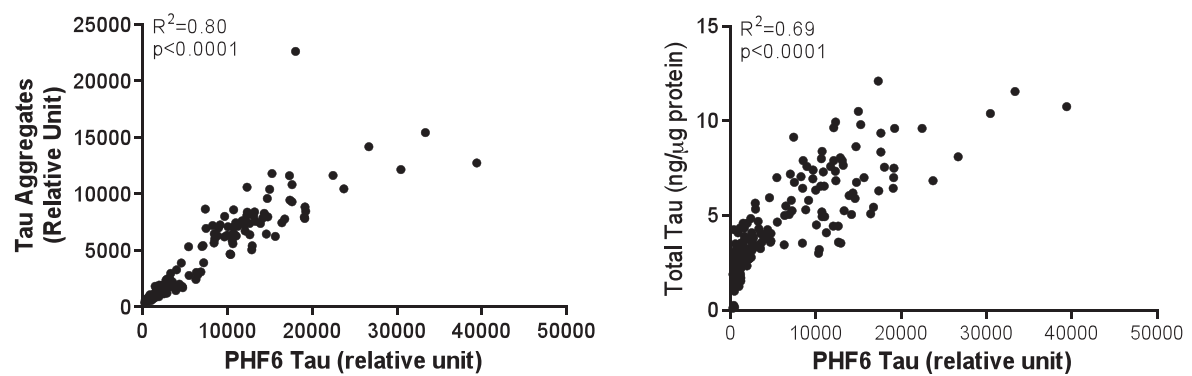

Figure 4 Correlation of various tau species in the insoluble fraction of $\mathbf{r T g} 4510$ mouse brain. The data in Figures 2 and 3 were analyzed for correlation with PHF6 tau using Prism 5.0 software. Shown are correlations of PHF6 tau with: A. AT8 tau; B. pThr tau global phosphorylation; C. tau aggregates; and $\mathbf{D}$. total tau.
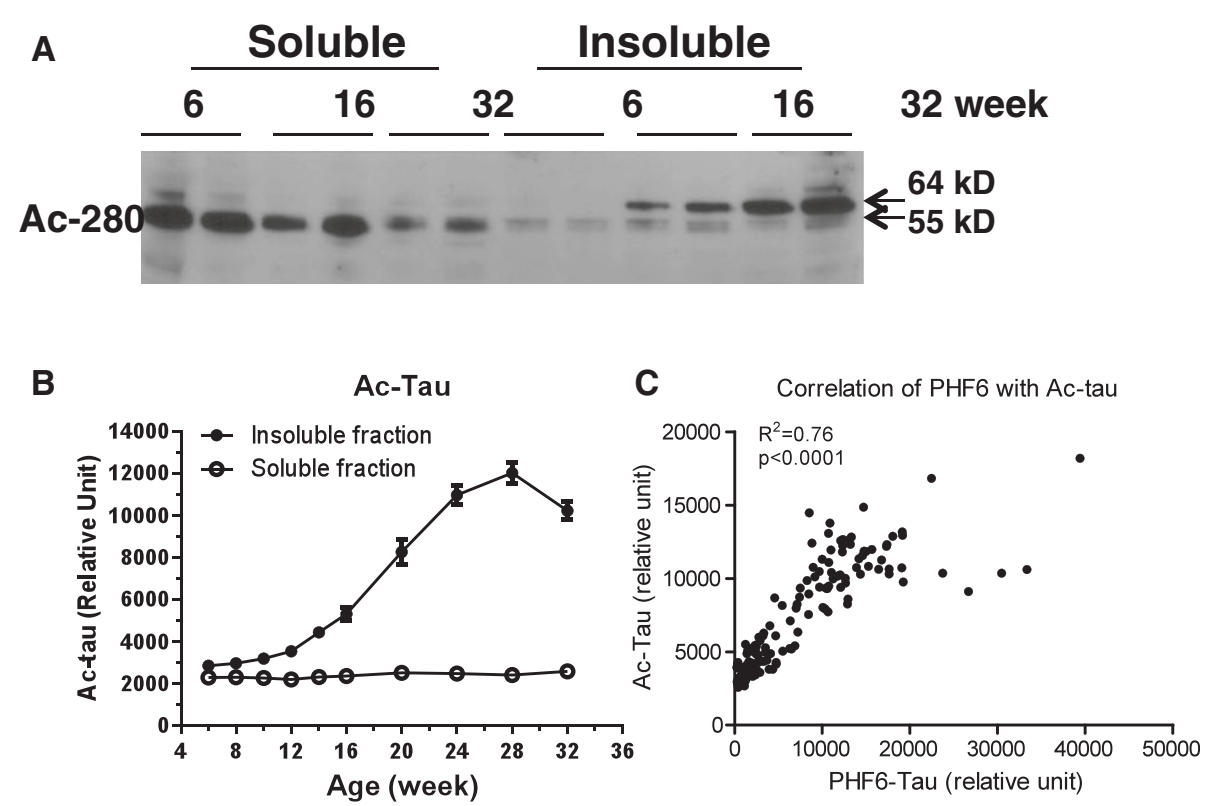

Figure 5 Acetylated tau in rTg4510 brain. A. Western blot of acetylated tau probed by ac-280, a polyclonal antibody specific for tau acetylated at Lys280. B. Age-dependent change of acetylated tau in the soluble and insoluble fractions of rTg4510 mouse brain. Acetylated tau was measured using $1 \mu \mathrm{g}$ of protein from the soluble or insoluble fractions of rTg4510 mouse brain with biotin HT7 for capture and a pan acetyl-lysine antibody for detection. The error bars represent the standard error for each group. The change of ac-tau reached statistical significance at 14-weeks of age as compared to 8-weeks ( $p<0.05$, one-way ANOVA). C. Correlation of the levels of acetylated tau and PHF6 tau in the insoluble fraction of rTg4510 mouse brain. 


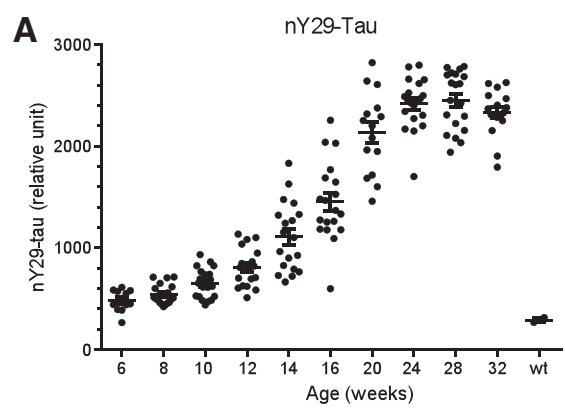

C Correlation of $\mathrm{nY} 29$ tau with PHF6 tau

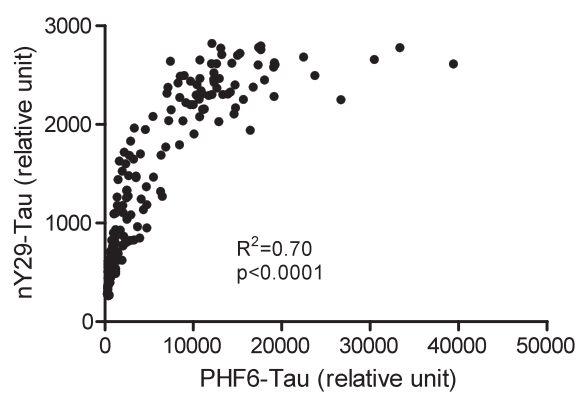

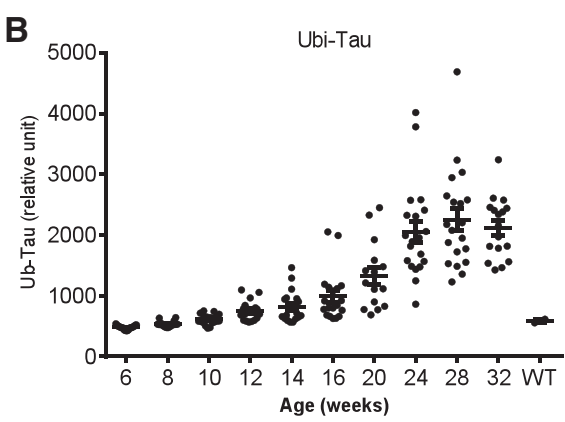

D

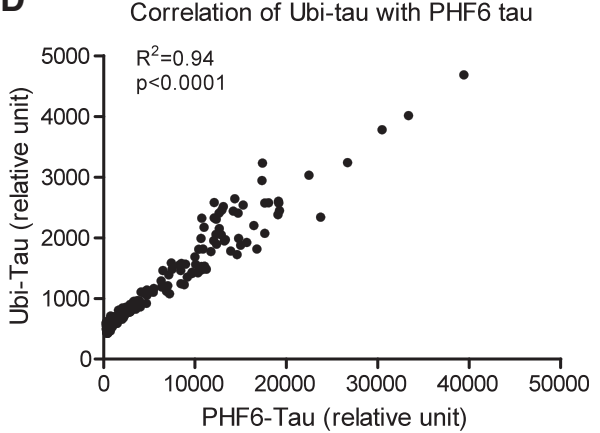

Figure 6 Age-dependent change of ubiquitination and nitration of tau in the brain insoluble fraction of rTg4510 mice. Post-translational modifications were measured using AlphaLISA immunoassays with $1 \mu \mathrm{g}$ of protein from the brain insoluble fraction of rTg4510 mice. A. Age-dependent change of tau ubiquitination. Ub-tau was detected using biotin $\mathrm{HT}-7$ for capture and an anti-ubiquitin antibody for detection. B. Age-dependent change of Tyr29 nitration of tau. nY29 tau was detected using biotin HT-7 for capture and an anti-nY29 antibody for detection. C. and D. Correlation of Ub-tau (C) and nY29 tau (D) with PHF6 tau. Correlation analysis was done using Prism 5.0 software.

Some of the correlations between different posttranslationally modified forms of tau, although significant, are not linear. This is likely due to the technical limitation that we cannot assess assay linearity without a standard for each tau species. Therefore, the sample loadings in the analyses were determined by dilution linearity of the middle-aged samples and thus may not cover the wide range of tau levels in the different age groups. The lack of assay standards for each specific tau species also means we cannot determine the absolute concentration and stoichiometry of each species in this pool of pathological tau. However, this limitation does not impact the conclusion that all post-translational modifications of tau studied are all temporally associated with the development of tauopathy.

Elevation of CSF total tau is a characteristic feature of $\mathrm{AD}$ and is being explored as a diagnostic biomarker and as a biomarker to identify patients in clinical trials of potential AD therapeutics [6]. The elevation of CSF tau was first hypothesized to be a consequence of neuronal
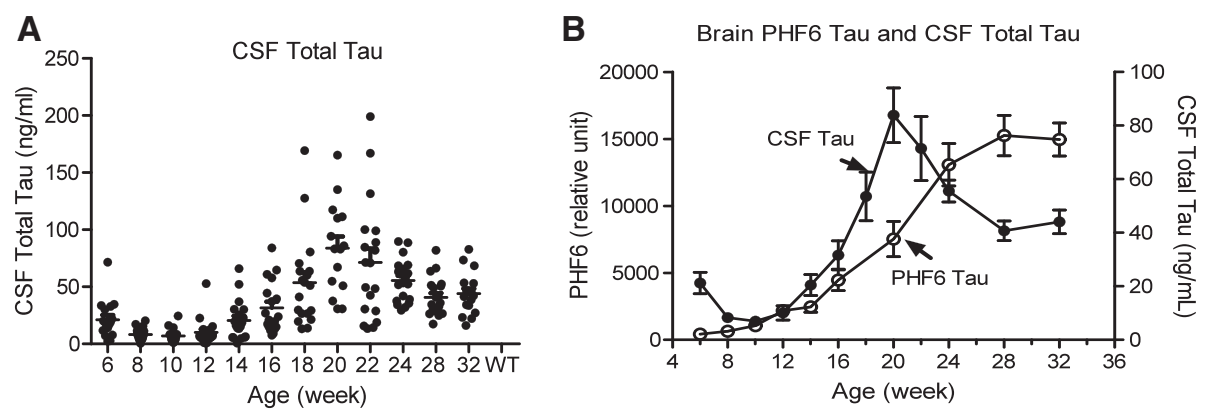

Figure 7 Age-dependent change of CSF total tau in rTg4510 mice. CSF total tau was measured using an AlphaLISA assay with biotin HT7 antibody for capture and antibody BT2 conjugated acceptor beads for detection. One microliter of CSF was used per assay and recombinant tau (Milipore) was used to generate a standard curve for quantification. A. Age-dependent change in CSF total tau in Tg4510 mice. B. Re-plotting of age-dependent changes in mean brain PHF6 tau levels from Figure 3A (open circle) and mean CSF tau levels from Figure 7A (solid circle). Error bars represent the standard error of the mean. 
A

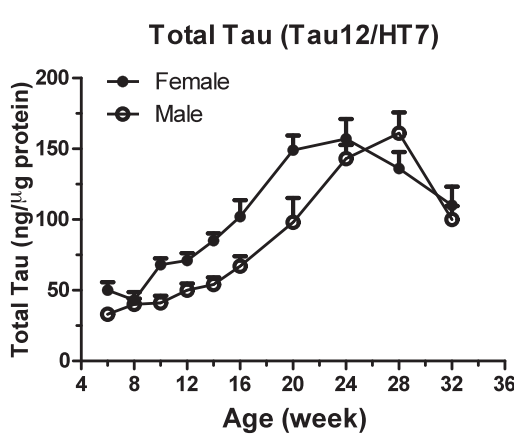

C

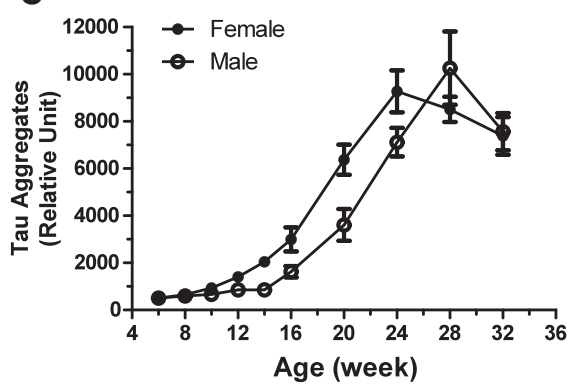

B

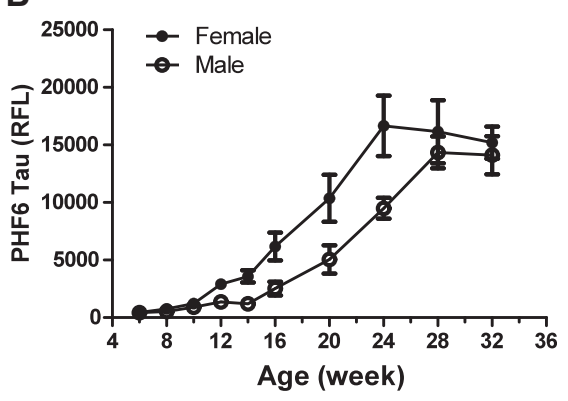

D

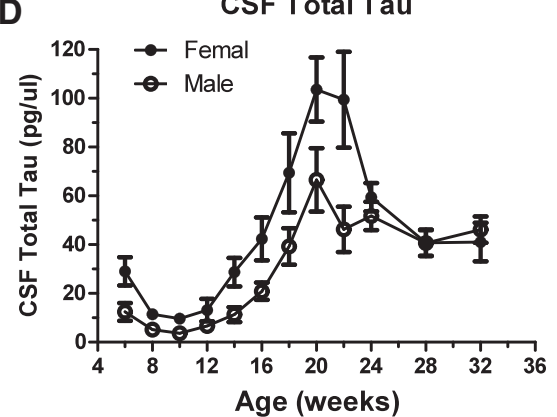

Figure 8 Gender difference in rTg4510 mice. Data from previous figures were plotted separately for male (open circle) and female (solid circle) mice. Error bars represent the standard error of the mean. Factorial ANOVAs were used to analyze the brain and CSF tau data for male and female mice at various ages with sex and age as the between subjects factors. A. Total Tau. Total tau levels were greater in the brains of female mice compared to males [main effect of sex, $F(1,166)=19.43, p<0.0001$, total tau levels varied as the mice aged and the age-related variation was different for females compared to males [Sex $x$ Age interaction, $F(9,166)=2.43, p<0.02$ ] with the males catching up to the females by 24 weeks of age. B. PHF6 Tau. Female mice had higher levels of PHF6 tau compared to males [main effect of Sex, F $(1,172)=18.78, p<0.0001$ ] and PHF6 tau levels increased as the mice aged. C. Tau Aggregation. Female mice showed greater tau aggregation than males [main effect of Sex, $F(1,170)=6.59, p<0.02]$, tau aggregation increased and peaked as the mice aged and the age-related variation was different for females compared to males [Sex $x$ Age interaction, $F(9,170)=2.56, p<0.009$ ] with the tau aggregation peaking earlier in females than males $(24$ weeks vs. 28 weeks). D. CSF Total Tau. Female mice had higher CSF total tau levels compared to males [main effect of Sex, F(1,197) $=21.24, p<0.0001$ ], CSF total tau levels varied as the mice aged and the difference between females and males varied at different ages [Sex $x$ Age interaction, $F(11,197)=2.31, p<0.02$ ] with similar levels being observed in females and males at older ages.

cell death, but this hypothesis is unlikely to explain the presence of tau in the CSF of healthy individuals of all ages. The emerging evidence that tau is released from normal cells and neurons [20-22], possibly as a mechanism of removing excessive cellular metabolites [23], provides an alternative hypothesis of the source of CSF tau. In rTg4510 mice, CSF tau changes in several phases as the animals age, a temporal pattern that is distinct from the steady increase of brain tauopathy (Figure 7B). Our findings are consistent with a previous report [24] and suggest that CSF tau levels in this model are likely determined by both normal tau metabolism (e.g. constitutive secretion by cells) and by processes associated with pathological tau formation. Further understanding the nature of age-dependent changes in CSF tau may lead to important insights into the mechanism of tauopathy progression and biomarker development for AD therapy.

Although there is a clear trend of pathological tau development in $\operatorname{Tg} 4510$ mice, the accumulation of pathological tau displays large variations in all parameters assessed. These variations are consistent with findings by others and are not due to differential transgene expression ([24], L. Song and L. Zhang unpublished data). It has been reported previously that female rTg4510 mice display more robust tau pathology and more severe deficits in spatial learning and memory tests [25]. Our data provide further evidence for a gender difference in that female $\mathrm{rTg} 4510$ mice showed significantly more aggressive age-dependent accumulation of pathological tau in all features we assessed (Figure 8A-D). The underlying mechanism for the gender difference is not clear but our observations provide important information for using this model in terms of gender selection or balance and group size to give sufficient power for target effect size.

\section{Conclusion}

Our data demonstrate that tauopathy in $\mathrm{rTg} 4510$ mice develops via accumulation of a pool of pathological tau that carries multiple post-translational modifications, a process that can be detected well before the histological detection of NFTs and is more pronounced in female rTg4510 mice. Although hyperphosphorylation is the best known 
characteristic of tauopathy, there are several additional post-translationally modified forms of tau (e.g. acetylation, ubiquitination, nitration) that correlate equally well with formation of pathological tau and also temporally correlate with formation of hyperphosphorylated tau. Therefore, it is not clear which of the post-translational modification events is the proximal cause of pathological tau formation. Nevertheless, the data suggests that a treatment that reduces pathological tau formation should lead to a correlative reduction of all tau species associated with tauopathy. CSF tau in rTg4510 mice is likely derived from both normal metabolism and the formation of pathological tau and exhibits a complex temporal pattern that is distinct from the progressive increase in pathological tau formation in the brain. Further understanding of the biological and pathological processes of CSF tau production remains an important aspect for future studies given its importance as a translational biomarker of efficacy and patient selection for preclinical and clinical testing of potential treatments targeting tauopathies. In summary, this study provides several important insights into the development of tauopathy in rTg4510 mice and into the use of this model to assess potential therapeutic treatments targeting tau pathology.

\section{Methods}

\section{Reagents}

Antibodies used for Western blots and AlphaLISA assays are listed in Table 2. Ac-280 is a custom rabbit polyclonal antibody developed against an acetylated tau peptide as

Table 2 Antibody reagents used in this study

\begin{tabular}{lll}
\hline Antibody & Epitope & Source \\
\hline HT7 & aa159-163 & Thermo Scientific \\
BT2 & aa194-198 & Thermo Scientific \\
Tau12 & N-terminus & Covance \\
AT270 & pT181 & Thermo Scientific \\
AT180 & pT231 & Thermo Scientific \\
AT100 & pS212/T214 & Thermo Scientific \\
PHF6 & PHF around pT231 & Covance \\
AT8 & pS202/T205 & Thermo Scientific \\
PHF13 & PHF pT396 & Covance \\
PS400 & phospho-S400 & Thermo Scientific \\
PS404 & phospho-S404 & Invitrogen \\
PS409 & phospho-S409 & Invitrogen \\
PS412 & phospho-S412 & AnaSpec \\
P-Thr & pan phosphor-threonine & Sigma \\
Ac-Lys & pan acetyl-lysine & Cell Signaling \\
Ac-280 & aa264-287 with acetyl-Lys280 & Prepared as described \\
& & in [13] \\
Ubiqutin & pan ubiquitin & Millipore \\
nY29 & nitrated tyrosine 29 & Abcam \\
\hline
\end{tabular}

described [14]. The serum was pre-absorbed with an unmodified tau peptide of the same amino acid sequence before being affinity purified using the acetylated peptide (GenScript). The specificity of ac-280 was confirmed by Western blots showing that this antibody selectively picked up hyper-acetylated tau when tau was co-transfected with histone acetyltransferase P300 in HEK293 cells (Additional file 3: Figure S3).

\section{Animals}

The rTg4510 mouse line overexpressing the human tau ON4R isoform carrying the P301L mutation [10] was licensed from the Mayo Clinic and bred at Taconic in Germantown, NY. The mice were bred by crossing the hTau responder line (FVB/N strain) and the tTA activator line (129S6 strain) as described [10]. All animal procedures are in compliance with the NIH Guide for the Care and Use of Laboratory Animals and were approved by the Institutional Animal Care and Use Committee of Merck Research Laboratories, an AAALAC accredited institution.

Animals of different ages (gender balanced, $n=18-20$ per group with the exception of 12 at 6 weeks of age and 15 at 20 weeks of age) were euthanized with $\mathrm{CO}_{2}$. Brain and CSF were collected from each animal. For CSF collection, mice were euthanized and placed on a table with the head tilted down 70-80 degrees. The skin was removed to expose muscles located between the ears, the caudal part of head and the rostral neck to locate the cisterna magna. A $27 \mathrm{G}$ butterfly needle connected to a $1 \mathrm{ml}$ syringe was used to punch through the muscle to the cisterna magna and about $10 \mu \mathrm{L}$ CSF was collected by slightly pulling the syringe.

\section{Preparation of brain fractions}

The soluble and insoluble fractions of $\mathrm{rTg} 4510$ mouse brain were prepared based on a previously published method with some modifications [12]. Briefly, half brains from rTg4510 or wild-type mice were homogenized using metal beads with TissueLyzer (Qiagen) in $900 \mu \mathrm{L}$ preparation buffer containing $50 \mathrm{mM}$ Tris, $\mathrm{pH} 8.0,250 \mathrm{mM}$ $\mathrm{NaCl}, 5 \mathrm{mM} \mathrm{KCl}, 2 \mathrm{mM}$ EDTA, $2 \mathrm{mM}$ EGTA, PhosphoSafe extraction buffer (EMD/Novagen) plus Complete EDTA-free Protease Inhibitor Tablet (Roche), PhoStop Tablet (Roche), $2 \mu \mathrm{M}$ Trichostatin A (Sigma-Aldrich), $5 \mathrm{mM}$ Nicotinamide (Sigma-Aldrich), and $1 \mu \mathrm{M}$ PUGNAc (Sigma-Aldrich). The homogenates were centrifuged at $14,000 \mathrm{~g}$ for $15 \mathrm{~min}$ to remove the tissue debris. The supernatants were then centrifuged at $100,000 \mathrm{~g}$ for $30 \mathrm{~min}$. The pellets were re-suspended in the preparation buffer, and the supernatants and the pellets from the second spin were defined as the soluble and insoluble fraction, respectively. Protein concentrations were determined using the $\mathrm{BCA}$ assay kit (Pierce). 


\section{AlphaLISA-based sandwich immunoassays}

A comprehensive panel of sandwich immunoassays listed in Table 1 was developed to measure different forms of tau. In general, analytes were captured by a monoclonal antibody that was biotinylated and bound to streptavidin coated donor beads (PerkinElmer). Detection was accomplished either by a monoclonal antibody conjugated to the acceptor beads directly or by a polyclonal antibody in combination with anti-rabbit IgG-conjugated acceptor beads (PerkinElmer).

Assay reactions $(25 \mu \mathrm{l})$ were carried out in OptiPlate384 microplates (PerkinElmer) that contained $5 \mu \mathrm{L}$ of analyte at the specified protein concentration, $10 \mu \mathrm{L}$ of biotinylated capture antibody (final concentration $2 \mathrm{nM}$ ) and $10 \mu \mathrm{L}$ of detection antibody-conjugated acceptor beads (final concentration $20 \mu \mathrm{g} / \mathrm{mL}$ ). After overnight incubation at $4^{\circ} \mathrm{C}, 25 \mu \mathrm{L}$ of streptavidin donor beads were added under subdued light conditions (final concentration $40 \mu \mathrm{g} / \mathrm{mL}$ ) and the reactions were incubated at room temperature for $60 \mathrm{~min}$ with gentle shaking. The fluorescent signal was detected on an Envision Plate Reader at $615 \mathrm{~nm}$ (PerkinElmer).

\section{Total tau and tau aggregates}

Total tau assays were defined as detection of tau using antibodies recognizing epitopes not affected by phosphorylation. Here we used biotin-Tau 12 with HT7 conjugated acceptor beads for analyzing brain total tau, and biotinHT7 with BT2 conjugated acceptor beads for analyzing CSF total tau. Recombinant tau (Millipore) was used to generate a standard curve for quantification. In addition, biotin-HT7 was also used in combination with HT7 conjugated acceptor beads for analyzing aggregated forms of tau. This assay detects any tau aggregate that is a dimer or larger, and thus does not distinguish between soluble oligomeric and insoluble, higher order aggregates of tau. Formic acid treatment, which denatures tau aggregates, abolished the signal in this HT7-HT7 assay (data not shown). The total tau assay detects both 55 and $64 \mathrm{kD}$ tau species while the aggregated tau assay preferentially detects the $64 \mathrm{kD}$ species that is enriched in aggregated forms of tau.

\section{Phospho-tau (p-tau)}

P-tau species were determined using biotin-HT7 as the capture antibody and acceptor beads conjugated to epitope-specific monoclonal antibodies for detection. Global phosphorylation was assessed by using biotin-HT7 as the capture antibody and a pan anti-pThr antibody conjugated to acceptor beads for detection. As there is no standard available for each p-tau and other post-translationally modified tau species, we cannot determine the absolute linearity for each assay. The dilution linearity was tested using the brain lysates of the 16 week old brain samples (data not shown) and the results were used to guide the amount of protein used in the assays.

\section{Acetylated tau (ac-tau), ubiquitinated tau (ub-tau) and nitrated tau (nY-tau)}

To assess tau acetylation, ubiquitination and nitration during aging, biotin-HT7 was used as the capture antibody in combination with either a pan anti-acetylated lysine monoclonal antibody, an anti-ubiquitin antibody or an anti-nY29 tau antibody conjugated to acceptor beads for detection.

\section{Western blot analysis}

The soluble or insoluble fractions of brain extracts were separated on NuPage gels (Invitrogen) and transferred to a nitrocellulose membrane. The antibody used in each blot was as specified. The blots were developed using electrochemiluminescence (ECL) according to the manufacturer's instruction (Amersham GE HealthCare).

\section{Statistical analysis}

The GraphPad Prism program was used for statistical analysis. Factorial ANOVAs were used to analyze the brain and CSF tau data for male and female mice at various ages with sex and age as the between subjects factors.

\section{Additional files}

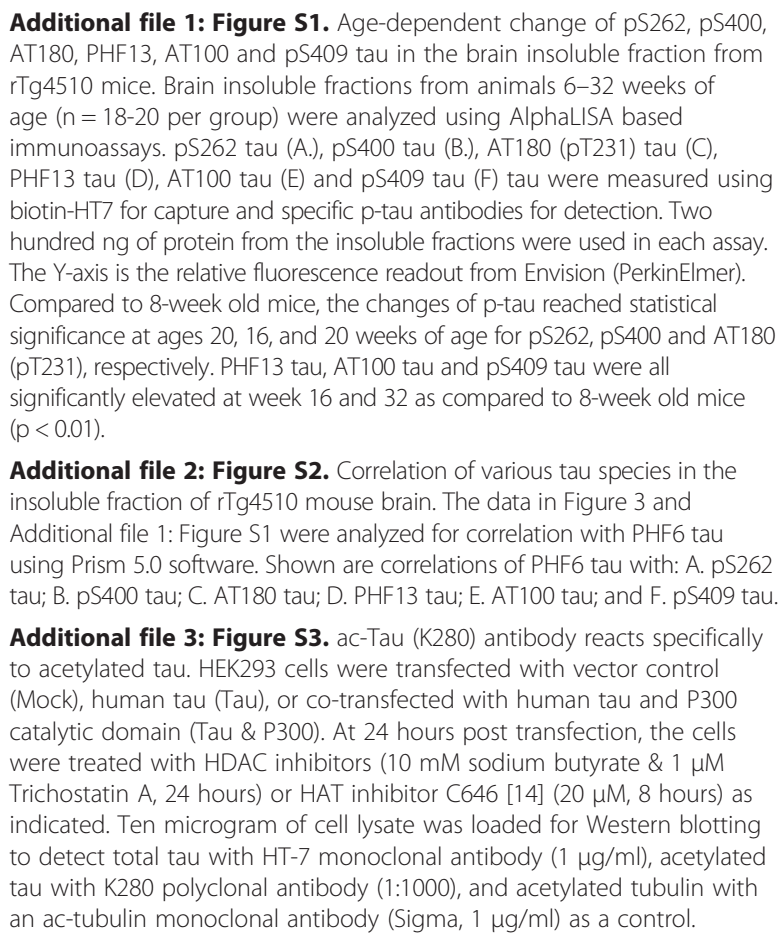

Additional file 2: Figure S2. Correlation of various tau species in the insoluble fraction of rTg4510 mouse brain. The data in Figure 3 and Additional file 1: Figure S1 were analyzed for correlation with PHF6 tau using Prism 5.0 software. Shown are correlations of PHF6 tau with: A. pS262 tau; B. pS400 tau; C. AT180 tau; D. PHF13 tau; E. AT100 tau; and F. pS409 tau.

Additional file 3: Figure S3. ac-Tau (K280) antibody reacts specifically to acetylated tau. HEK293 cells were transfected with vector control (Mock), human tau (Tau), or co-transfected with human tau and P300 catalytic domain (Tau \& P300). At 24 hours post transfection, the cells were treated with HDAC inhibitors (10 mM sodium butyrate \& $1 \mu \mathrm{M}$ Trichostatin A, 24 hours) or HAT inhibitor C646 [14] (20 $\mu \mathrm{M}, 8$ hours) as indicated. Ten microgram of cell lysate was loaded for Western blotting to detect total tau with HT-7 monoclonal antibody $(1 \mu \mathrm{g} / \mathrm{ml})$, acetylated tau with K280 polyclonal antibody (1:1000), and acetylated tubulin with an ac-tubulin monoclonal antibody (Sigma, $1 \mu \mathrm{g} / \mathrm{ml}$ ) as a control.

\section{Abbreviations}

AD: Alzheimer's disease; FTDP-17: Frontotemporal dementia with parkinsonism on chromosome 17; NFT: Neurofibrillary tangles; CSF: Cerebrospinal fluid; p-tau: Phosphorylated tau. 


\section{Competing interests}

All authors were employees of Merck Research Laboratories at the time of the study.

\section{Authors' contributions}

$L S, S X L, X O, J M, J L, G T$ performed the experiments and data analysis. LS, XW, $\mathrm{LH}, J F H, E M P$ and $L Z$ contributed to the study design, data analysis and writing the manuscript. All authors read and approved the final manuscript.

\section{Author details}

'Department of Neuroscience, Merck Research Laboratories, Kenilworth, NJ, USA. ${ }^{2}$ Molecular Biomarkers, Merck Research Laboratories, Kenilworth, NJ, USA. ${ }^{3}$ In Vivo Pharmacology, Merck Research Laboratories, West Point, PA, USA. ${ }^{4}$ Current address: BioDuro, a PPD Company, Beijing, China. ${ }^{5}$ Current address: Medical Oncology Department, Rutgers Cancer Institute of New Jersey, New Brunswick, NJ, USA. ${ }^{6}$ Current address: Human Oncology and Pathogenesis Program, Memorial Sloan Kettering Cancer Center, New York, NY, USA. ${ }^{7}$ Current address: Department of Neuroscience, Novartis Institute for Biomedical Research, Cambridge, MA, USA.

Received: 5 April 2014 Accepted: 2 March 2015

Published online: 26 March 2015

\section{References}

1. Mandelkow EM, Mandelkow E. Biochemistry and cell biology of tau protein in neurofibrillary degeneration. Cold Spring Harb Perspect Med. 2012;2: a006247.

2. Lee VM, Goedert M, Trojanowski JQ. Neurodegenerative tauopathies. Ann Rev Neurosci. 2001;24:1121-59.

3. Selkoe DJ. Alzheimer's disease: genes, proteins, and therapy. Physiol Rev. 2001;81:741-66.

4. Martin L, Latypova X, Terro F. Post-translational modifications of tau protein: Implications for Alzheimer's disease. Neurochem Int. 2011;58:458-71.

5. Avila J, Lucas JJ, Perez M, Hernandez F. Role of tau protein in both physiological and pathological conditions. Physiol Rev. 2004;84:361-84.

6. Blennow K, Hampel H, Weiner M, Zetterberg H. Cerebrospinal fluid and plasma biomarkers in Alzheimer disease. Nat Rev Neurol. 2010;6:131-44.

7. Goedert M, Jakes R. Mutations causing neurodegenerative tauopathies. Biochim Biophys Acta. 2005;1739:240-50.

8. Hutton M, Lendon CL, Rizzu P, Baker M, Froelich S, Houlden H, et al. Association of missense and $5^{\prime}$-splice-site mutations in tau with the inherited dementia FTDP-17. Nature. 1998;393:702-5.

9. Götz J, Deters N, Doldissen A, Bokhari L, Ke Y, Wiesner A, et al. A decade of tau transgenic animal models and beyond. Brain Pathol. 2007;17:91-103.

10. SantaCruz K, Lewis J, Spires T, Paulson J, Kotilinek L, Ingelsson M, et al. Tau suppression in a neurodegenerative mouse model improves memory function. Science. 2005:309:476-81.

11. Ramsden M, Kotilinek L, Forster C, Paulson J, McGowan E, SantaCruz K, et al. Age-dependent neurofibrillary tangle formation, neuron loss, and memory impairment in a mouse model of human tauopathy (P301L). J Neurosci. 2005;25:10637-47.

12. Berger Z, Roder H, Hanna A, Carlson A, Rangachari V, Yue M, et al. Accumulation of pathological tau species and memory loss in a conditional model of tauopathy. J Neurosci. 2007;27:3650-64.

13. Cohen TJ, Guo JL, Hurtado DE, Kwong LK, Mills IP, Trojanowski JQ, et al. The acetylation of tau inhibits its function and promotes pathological tau aggregation. Nat Commun. 2011;2:252.

14. Min SW, Cho SH, Zhou Y, Schroeder S, Haroutunian V, Seeley WW, et al. Acetylation of tau inhibits its degradation and contributes to tauopathy. Neuron. 2010;67:953-65.

15. Cripps D, Thomas SN, Jeng Y, Yang F, Davies P, Yang AJ. Alzheimer disease-specific conformation of hyperphosphorylated paired helical filament-Tau is polyubiquitinated through Lys-48, Lys-11, and Lys-6 ubiquitin conjugation. J Biol Chem. 2006;281:10825-38.

16. Reynolds MR, Reyes JF, Fu Y, Bigio EH, Guillozet-Bongaarts AL, Berry RW, et al. Tau nitration occurs at tyrosine 29 in the fibrillar lesions of Alzheimer's disease and other tauopathies. J Neurosci. 2006;26:10636-45.

17. Reyes JF, Fu Y, Vana L, Kanaan NM, Binder LI. Tyrosine nitration within the proline-rich region of Tau in Alzheimer's disease. Acta Neuropathol. 2012;123:119-32
18. Spires TL, Orne JD, SantaCruz K, Pitstick R, Carlson GA, Ashe KH, et al Region-specific dissociation of neuronal loss and neurofibrillary pathology in a mouse model of tauopathy. Am J Pathol. 2006;168:1598-607.

19. Sahara N, DeTure M, Ren Y, Ebrahim AS, Kang D, Knight J, et al. Characteristics of TBS-extractable hyperphosphorylated tau species: aggregation intermediates in rTg4510 mouse brain. J Alzheimer's Dis. 2013;33:249-63.

20. Yamada $K$, Cirrito JR, Stewart FR, Jiang H, Finn MB, Holmes BB, et al. In vivo microdialysis reveals age-dependent decrease of brain interstitial fluid tau levels in P301S human tau transgenic mice. J Neurosci. 2011;31:13110-7.

21. Pooler AM, Phillips EC, Lau DH, Noble W, Hanger DP. Physiological release of endogenous tau is stimulated by neuronal activity. EMBO Rep. 2013:14:389-94.

22. Kim W, Lee S, Hall GF. Secretion of human tau fragments resembling CSF-tau in Alzheimer's disease is modulated by the presence of the exon 2 insert. FEBS Lett. 2010;584:3085-8.

23. Gendreau KL, Hall GF. Tangles, toxicity, and tau secretion in AD - new approaches to a vexing problem. Front Neurol. 2013;4:160.

24. Barten DM, Cadelina GW, Hoque N, DeCarr LB, Guss VL, Yang L, et al. Tau transgenic mice as models for cerebrospinal fluid tau biomarkers. J Alzheimer's Dis. 2011;24 Suppl 2:127-41.

25. Yue M, Hanna A, Wilson J, Roder H, Janus C. Sex difference in pathology and memory decline in rTg4510 mouse model of tauopathy. Neurobiol Aging. 2011;32:590-603.

\section{Submit your next manuscript to BioMed Central and take full advantage of:}

- Convenient online submission

- Thorough peer review

- No space constraints or color figure charges

- Immediate publication on acceptance

- Inclusion in PubMed, CAS, Scopus and Google Scholar

- Research which is freely available for redistribution 